\title{
Tesis de economía 1990
}

1. "Dialéctica de la Concepción Económica Neoliberal en El Salvador". Carlos Fidel Hernández Moreira.

Febrero 1990.

Las grandes temáticas que se abordan son las siguientes: Análisis de la derecha tradicional y nueva derecha en E.U.A. con su proyecto para el tercer mundo. Análisis del origen de la derecha tradicional y nueva derecha en El Salvador; analizando la viabilidad del proyecto de ésta última.

2. "La intervención del Estado en la Economia Salvadorefía en el período 1970-88 y la privatización de las Empresas de CORSAIN" Celso Ricardo Coto Romero, Mildred lliana Cárcamo, José Antonio Recinos Díaz.

Marzo 1990.

Se parte de la visión neo-liberal de la participación del Estado en la Economia y de experiencias de privatización en Inglaterra y Chile. Luego se subdivide el periodo para 1970-79 y 1980-88 haciendo para cada periodo un estudio comparativo de los sectores privado y público (inversión, consumo, empleo, presión fiscal, gasto público-PIB); desarrollo de las empresas estatales de cada periodo. Finalmente se ofrece un análisis financiero, de generación de empleo y participación en el mercado de las empresas administrativas por CORSAIN (Hotel Presidente, complejo Pesquero Industrial, Centro Gráfico, Ingenio Jiboa, Textilera Izalco).

3. "Evolución del Financiamiento externo en la Economia Salvadoreńa en el periodo 1978-1988 y su papel en la implementación de un Programa de Ajuste".

Merlin Alejandrina Barrera López, Ana Giadys Buendia Canizález, José Atilio Rodriguez Pacheco. 
Marzo 1990.

Se parte de una conceptualización y fuentes de Cooperación Externa, luego se sigue la evolución de los montos de cooperación externa por sectores económicos y sociales a través del Presupuesto Ordinario, Presupuesto Extraordinario (PERE) e Instituciones Autónomas para 1978-1988. Se examina el comportamiento de las principales variables macroeconómicas y sociales para el periodo. Finalmente se ofrece un marco conceptual de los Programas de Ajuste Estructural, experiencias de éstos en algunos paises de A.L. y un lineamiento estratégico para la implementación del PAE en el pais.

4. "Génesis y Contribución de la Asistencia Norteamericana a través del "Presupuesto extraordinario de Reactivación Económica" en la Coyuntura Salvadoreña de 1983-1988".

Rubia Rubenia Flores Jurado, Norma Elizabeth Molina López y Graciela Marta Villamariona Romero.

Septiembre 1990.

Se sigue la evolución de la crisis fiscal 1980-88; luego se detalla el origen del PERE, su estructura (Programa para la Reforma Agraria, generación de empleo, restauración de servicios básicos, asistencia humanitaria), negociación de los fondos GOES/AID (convenio apoyo de la balanza de pagos, PL-480, etc.), fuentes de financiamiento, leyes instructivos para el manejo de fondos. Finalmente se ve la evolución, destino y evaluación económica-política del PERE/ AID.

5. "El transporte mecanizado no capitalista de pasajeros en el área metropolitana de San Salvador".

José Emilio Márquez h. y Rolando Antonio Moreno Rodríguez.

Marzo 1990.

Contiene una visión global del pequeno transportista de pasajeros en el AMSS, un análisis teórico del proceso de trabajo del transporte capitalista y de las categorias de subsunción directa e indirecta del trabajo por el capital, a su vez detalla una serie de indicadores que acusan al TNCP en el E.S. como una forma de producción no capitalista que se encuentra subsumida indirectamente al capital.

6. "Industrialización y Desarrollo Tecnológico en El Salvador 19501987".

Rosa Maria Mendoza Hidalgo y Rosalinda del Cid de Fernández. Abril 1990.

Se realiza un marco teórico sobre el desarrollo y la tecnologia para luego estudiar la evolución de la inversión extranjera directa, impor- 
taciones y capacitación de recursos humanos en las etapas de inicio de la industria, integración centroamericana, fomento de exportaciones en los 60's y en la crisis de los 80 's.

7. "Las relaciones económicas con terceros países: 1970-1987". Rhina Elizabeth Jiménez, Regina Elizabeth Ventura y Angel Mauricio Villalobos.

Abril 1990.

Se trabaja con dos periodos $1970-79$ y 1980-87, analizando para ambos las principales políticas para el sector externo; la dinámica de las relaciones comerciales en mercados tradicionales y no-tradicionales; evolución de la deuda externa y fuentes de financiamiento.

8. "Política monetaria en El Salvador: Evaluación de la coherencia interna y represión financiera. Periodo 1960-1988".

Héctor Ivan Borja y Eduardo Osmín Rodríguez.

Mayo 1990.

Se define el papel, objetivos e instrumentos de la política monetaria. Se retoma el marco legal e institucional que ha enmarcado a dicha política en E.S. para 1960-1988. Se realiza un seguimiento de los objetivos e instrumentos de la política para dicho período. Finalmente se hace una evaluación de la eficacia de ésta.

9. "Incidencias de las políticas comercial e industrial en el desarrollo del aparato productivo industrial salvadorefio y sus efectos en el conjunto de sectores económicos 1960-1988".

Margarita Sibrián Echeverria, Juan Ricardo Sánchez y Jesús Antonio Almendárez.

Agosto 1990.

Se parte de un análisis de las políticas comerciales (sobre todo el proteccionismo) y su impacto en la industrialización. Se analiza los avances en el proceso de sustitución de importaciones; en el carácter monopólico y oligopólico del sector; los factores que limitan su crecimiento (estrechez de mercado, dependencia tecnológica). Finalmente se ofrece una metodología para el cálculo de los traslados del excedente en la economía entre los diversos sectores en la evolución real y corriente.

10. "Análisis de las metodologías utilizadas en la evaluación económica de proyectos de caminos rurales en El Salvador y metodología recomendada".

Ruth del Castillo Rodríguez, Carlos Armando Rivera Ramos y William Alberto Sanchez.

Agosto 1990. 
Enfoca de manera general la evaluación de proyectos y sus componentes, define lo que es un camino rural destacando la importancia del mismo en el desarrollo económico y social de la zona rural del pais, a su vez explica las metodologias utilizadas en la evaluación de proyectos en E.S., analizando si estas resultan apropiadas o no para la realidad del país.

11. "Análisis comparativo de la Banca Privada y Banca Nacionalizada en El Salvador: 1971-1988".

Ricardo Ernesto Granadino, Oscar Orlando Hernández y Antonio Roberto Arévalo Damas.

Septiembre 1990.

Luego de presentar la visión neo-liberal y estructural sobre el sistema bancario, se realiza el análisis comparativo a través de: La estructura del crédito (montos, por sector económico, por tamaño económico del usuario, tipo de garantia, plazos); mora y refinanciamiento; eficiencia (liquidez, solvencia, crecimiento, rentabilidad, costos, margenes de intermediación, productividad).

12. "La evolución del precio de la vivienda en el área metropolitana de San Salvador. (1973-1989)."

José Rigoberto Velásquez Wills, Joaquín Omar Benavides. Sept/ 90.

Como antecedentes se presenta la problemática habitacional del AMSS para el periodo 1960-1973 en cuanto a: población, disponibilidad de suelo, formas que asume el proceso de producción y comportamientos de los precios. Se analiza la conformación y componentes del precio de la vivienda: costo de mano de obra, suelo urbano, financieros, materiales de construcción, gastos administrativos y ganancia del capital. Finalmente se sigue el comportamiento de los componentes del precio para 1973-1989.

13. "La estrategia económica del Gobierno de ARENA: caracterización y perspectivas".

Nicolás Alfredo Martínez, Graciela Yaceny Nieves y José Angel Tolentino.

Septiembre 1990.

Se parte de un marco teórico sobre los programas de estabilización y de ajuste estructural; luego se analiza la naturaleza (causas coyunturales y estructurales) y dimensión de la crisis socioeconómica salvadorena; se examina la estrategia del gobierno de ARENA analizando su viabilidad técnica, financiera, política e institucional.

14. "Determinantes externos del crecimiento económico en El Salvador 1962-1988. Un modelo econométrico aplicado a la economia salva- 
dorena".

Ena Cuestas Duenas, Rafael Antonio Solózano y Alvaro Trigueros. Se estudia el modelo de las dos brechas, el enfoque keynesiano y el enfoque estructurallista con aporte de Kalecki sobre el crecimiento económico y la restricción externa. Luego se define y estima un modelo econométrico (incluye la función ahorro, inversión, importaciones, exportaciones, producción y flujo de capital extranjero), analizando sus resultados.

15. "Los mecanismos de pago intracentroamericanos: sus efectos en el comercio regional particularmente de El Salvador con el resto de paiíses del área (1960-1988)".

Xiomara Bernabe Portillo, José Antonio Hernández y José Manuel Iraheta.

Septiembre 1990.

Se parte de un análisis del proceso de integración monetaria centroamericana, para estudiar luego el sistema multilateral de pagos y el intercambio regional tanto en su fase óptima: 1962-1978 como en la etapa crítica: 1979-1988 destacando los elementos que la paralizaron. Se estudia los mecanismos coyunturales de pago para el periodo 1983-88 destacando sus alcances y limites. Finalmente se realiza un análisis estructural de los mecanismos de pago y se aportan gerencias para su mejoramiento y relanzamiento del comercio.

16. "Inserción de los migrantes en el mercado de trabajo del área metropolitana de San Salvador"

Mario Roger Hernández y Luis Ernesto Romano.

Septiembre 1990.

Se establecen un conjunto de factores determinantes de la migración interna y de las formas de inserción al mercado de trabajo. Se muestra la dinámica de la migración interna para 1950-1986. Luego con la utilización de encuestas se analiza la dinámica migracional y las modalidades de inserció al mercado de trabajo del AMSS para 1990.

17. "Elementos desencadenantes y amplificadores de la crisis de la caficultura salvadoreña 1970-1989".

José Calixto Arias, Carlos Armando Pineda y Carlo Fernando Trigueros.

Septiembre 1990.

Se dimensiona la importancia del café en la economia salvadoreña (como parte del producto, generador de divisas, empleo, salarios, ingresos fiscales); se compara la caficultura salvadorefía con la del 
área centroamericana y se analizan la causas estructurales (envejecimiento del parque cafetalero, técnicas de cultivo, etc) y los factores coyunturales (precios internacionales, guerra, plagas, política económica, etc) que amplificaron su crisis.

1991

1. "Incentivos económicos a la industria y a la agricultura de exportación tradicional durante el período 1960-1980".

Blanca Lidia Cerritos Chávez.

Enero 1991.

Contiene un estudio del sector industrial y el subsector cafetalero analizando y comparando las políticas de desarrollo implementadas en ambos, evaluando el grado en que dichas políticas contribuyeron o no al nivel de industrialización alcanzado, estableciendo que sector ofreció más ventajas de inversión y cuál era sujeto de políticas de fomento estatal. A su vez se analiza elpapel de las politicas de fomento dentro de la actual estrategia de gobierno.

2. "Crisis agricola y política agraria en El Salvador en la década de los ochenta.Tendencias y perspectivas".

Rolando Almendárez, Serafín Cardoza y José Reyes Alfaro.

Marzo 1991.

Contiene un análisis de la vinculación política agraria y desarrollo económico, además se estudia el origen y dimension de la crisis agricola en E.S. haciendo un examen de la política agraria aplicada en la década de los 80 s evaluando la influencia de la misma sobre la seguridad alimentaria y la producción altamente lucrativa, a su vez se analizan en forma breve la estrategia agraria del actual gobierno.

3. "Efectos de la aplicación de las medidas de liberalización financiera sobre el sistema de ahorro y préstamo".

Nurith Barrientos, Graciela Fontg y Angélica Maria Figueroa.

Abril 1991.

Se inicia revisando las visiones Neoliberales y del estructuralismo -keynesiano sobre el desarrollo económico. Se da una evolución histórica del sistema financiero salvadoreño. Se analiza la contribución de las Asociaciones de Ahorro y Préstamo (AA)) al financiamiento habitacional; el efecto de la liberalización financiera sobre las A.A.P. y evaluación de alternativas para su funcionamiento en el actual contexto de privatización y liberalización. 
4. "Efectos del flujo de divisas generado por las exportaciones de café en las principales variables macroeconómicas de la Economía salvadorena. Periodo 1960-1989".

Margarita del Rocio Cárdenas y Luis Felipe Marenco.

Julio 1991.

Se parte de los enfoques sobre el efecto de las exportaciones de café sobre la brecha del sector externo, ahorro-inversión. PIB y déficit fiscal. Se analiza el mercado mundial de café y finalmente se estima el modelo las dos brechas y se interpretan los resultados econométricos obtenidos.

5. "Análisis de la conveniencia de introducir un impuesto al valor agregado en EL Salvador".

Mauricio Chicas Monzón.

Julio 1991.

Los elementos que considera son: un detalle de los aspectos básicos sobre el IVA, mencionando ventajas, desventajas, objetivos, tasas aplicables, métodos de cálculo, etc.; un análisis de la estructura tributaria salvadorena y consideración sobre la evaluación financiera y macroeconómica del IVA, los efectos del mismo sobre la estructura tributaria de E.S. y la conveniencia de introducirlo a no en el pais.

6. "Repercusiones de las políticas de ajuste estructural sobre el mercado de trabajo urbano de la economía salvadorena 1990".

Mirena del Rosario Escobar y Daniel Flores.

Agosto 1991.

Se hace una caracterización de la crisis económica y un diagnóstico económetrico del mercado de trabajo estudiando la relación crisis-empleo-salario, a su vez se destacan las repercusiones de los PAE, sobre el empleo y salarios reales de los grupos urbanos pobres. También se destaca la informalización de la estructura ocupacional como respuesta del mercado de trabajo al PAE.

7. "El impacto de las políticas de estabilización económica sobre la satisfacción de las necesidades básicas en los sectores urbanos en situación de pobreza en El Salvador en 1989-1990".

Carlos Acevedo, Edgar Flores y Rolando Arévalo.

Septiembre 1991.

Se realiza un marco teórico sobre los Programas de estabilización y de Ajuste Estructural; se analiza la concepción estrategica del programa económico de ARENA; se estudia la evolución de la canasta básica de bienes y servicios y de los niveles de pobreza. Finalmente se define y estima la deuda social. 
8. "Implicaciones de los programas de ajuste estructural sobre pequehos productores de granos básicos en El Salvador".

Anabelle Machuca Machuca, Rafael Antonio Pleitez y Gloria Yanira Quiteno.

Septiembre 1991.

Se realiza un diagnóstico integral de los productores que incluye: un análisis histórico de la tenencia y usos de la tierra; el acceso a recursos (tierra, trabajo, capital, comercialización y tecnologia); tipologia y condiciones de vida de los productores; situación de la seguridad alimentaria; política macroeconómica y sectorial en los 80 's. Luego se analiza conceptualmente a los programas de estabilización y ajuste estructural; detallándose las principales medidas implementadas en el pais. Finalmente se analiza las implicaciones del PAE sobre los beneficios netos y producción de los productores, recomendándose una política alternativa para el subsector. 